\title{
Interrelations of gas exchange cycles, body movements and heartbeats in the foragers of bumblebee Bombus terrestris (Hymenoptera: Apidae) at low temperatures
}

\author{
Aare KUUSIK ${ }^{1 *}$, Ants-Johannes MARTIN ${ }^{1}$, MarIKa MÄND ${ }^{1}$, KÜlli HIIESAAR ${ }^{1}$, LuUle METSPALU ${ }^{1}$ and URMas \\ TARTES $^{2}$
}

${ }^{1}$ Institute of Plant Protection, Estonian Agricultural University, Kreutzwaldi 64, 51014 Tartu, Estonia

${ }^{2}$ Institute of Zoology and Botany, Estonian Agricultural University, Riia 181, 51014 Tartu, Estonia

Key words. Bombus terrestris, gas exchange cycles, body movements, heartbeats

\begin{abstract}
Rhythmic body movements, cyclic gas exchange and heart activity were studied at low temperature (mostly at $5^{\circ} \mathrm{C}$ ) in bumblebee Bombus terrestris foragers. Simultaneous measurements by means of an electrolytic respirometer combined with an optical system by infrared radiation, revealed a co-ordination between discontinuous gas exchange cycles and body rhythmic movements. No distinct correlation was found between intermittent heart activity and body movements. The carbon dioxide bursts were actively ventilated by abdominal vigorous pumping movements. These bursts followed each other with intervals of 25-30 minutes.

The periods of heart activity regularly alternated with heart pauses. The frequency of the heartbeat was similar to that of the weak abdominal pulsations $(0.2-0.25 \mathrm{~Hz})$ which were imperceptible with the naked eye. All bumblebees displayed abdominal pulsations which were independent of vigorous pumping movements. In bumblebee B. terrestris foragers haemolymph oscillation was assumed at $5^{\circ} \mathrm{C}$, as is known in the honeybee and some other Bombus species.
\end{abstract}

\section{INTRODUCTION}

Bumblebee workers often have to survive in temperatures near zero. So far little is known about the breathing patterns of bumblebees at low temperatures close to their cold stupor points.

In many insects respiration occurs by discontinuous gas exchange cycles or DGCs, which means that the carbon dioxide is released discontinuously by bursts. Commonly the DGC is devided into three phases according to the action of the spiracles: the "C phase" of closed spiracles, the flutter or "F phase" when only a little carbon dioxide is released but oxygen is consumed, and finally, there is the phase of opened spiracles or "O phase" when carbon dioxide is released in bursts (Kestler, 1984; Hadley, 1994; Lighton, 1994; Snyder et al., 1995).

Most data about the respiration of bumblebees concern that of queens at room temperatures (e.g. Kammer \& Heinrich, 1974; Silvola, 1984). The bumblebee B. terrestris queens display discontinuous gas exchange cycles in different phases of their life cycle at $18^{\circ} \mathrm{C}$ (Beekman \& Stratum, 1999). DGCs have also been described in the honey bee Apis mellifera (Rothe \& Nachtigall, 1989; Lighton \& Lovegrove, 1990).

The bumblebee workers are very active at room temperature and only the lowering of temperature allowed for examination of their respiratory patterns. The aim of the present paper was to study the possible coordination between gas exchange, abdominal movements and heartbeats in the foragers of bumblebee $B$. terrestris at $5^{\circ} \mathrm{C}$.

\section{MATERIAL AND METHODS}

The bumblebee B. terrestris colonies (Natupol hives) were purchased from Koppert Biological Systems and were hold in an orchard in Tartu, Estonia. Thirty individuals from three colonies were tested. The bumblebee colonies and individuals used in this study were not parasitized, nor infected by diseases.

For the experiments foragers weighing 220-260 mg were selected from the colony and adapted to $5^{\circ} \mathrm{C}$ for one day before laboratory measurements.

Metabolic rate, discrete $\mathrm{CO}_{2}$ releases and body movements were recorded by means of an electrolytic differential microrespirometer, where the consumed oxygen was replaced continuously by adjusting the level according to pressure changes in the insect chamber (for more details see Kuusik et al., 1991, 1996; Tartes et al., 1999, 2000). In the present modification of this respirometer, standard plastic tubes (Eppendorf, volume of $1.5 \mathrm{ml}$ ) were used as insect chambers, and were connected to the respirometric system by rubber tubing (inner diameter $0.5 \mathrm{~mm}$ ).

The units on the graphs " $\mathrm{VO}_{2}\left(\mathrm{ml} \mathrm{h}^{-1}\right)$ " represent the rate of the production of oxygen by electrolysis. Using a personal computer the mean metabolic rate was calculated by averaging data over a longer period involving at least 3 DGCs (see Tartes et al., 2000).

The respirometer was combined with an infrared (IR) optical device. Two IR-emitting diodes were placed on the side of the chamber near to the ventral side of an insect. Two IR-sensitive sensors were placed on the opposite side of the chamber. Light from IR-diodes was modulated by the contractions of the heart and skeletal muscles. The output voltage level reflected the vigour of the muscular contractions of the insect.

We used 30 bumblebee individuals in our measurements. On the graphs the patterns of single individuals are represented, but these general patterns were also characteristic of all of the individuals studied.

\footnotetext{
* Corresponding author. E-mail: kuusiaar@eau.ee
} 


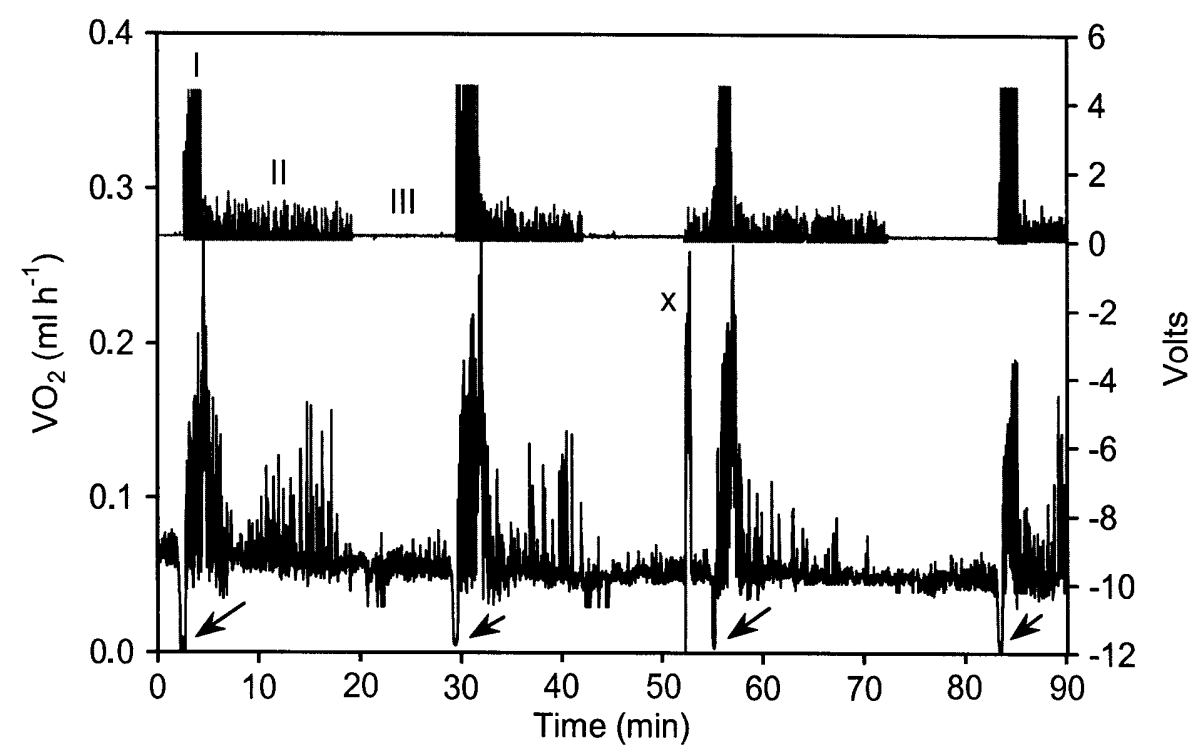

Fig. 1. Recording of IR-sensor (upper trace, Volts) and simultaneous respirogram (lower trace) showing cyclic gas exchange, abdominal movements and heart activity in a bumblebee Bombus terrestris forager. I - The period of abdominal pumping, together with other movements and heartbeats; II - the pattern due to abdominal pulsations mixed with heartbeats; III - heartbeats with very low amplitudes interpreted as heart pause. $\mathrm{CO}_{2}$ bursts are indicated with arrows. A body rotation stroke is seen $(\mathrm{x})$.

The analogue-to-digital conversions of the signals from the measuring apparatus were made by means of a data acquisition board DAS 1601 (Keithley-Metrabyte) with Test Point software (Capital Equipment Corp., Billerica, MA, U.S.A.). The sampling rate was usually $10 \mathrm{~Hz}$.

\section{RESULTS}

\section{Cyclic gas exchange}

The bumblebee workers exhibited clear cycles of discontinuous gas exchange while restrained and motionless. The immobility of bumblebees was achieved by exposing them to $5^{\circ} \mathrm{C}$; i.e., above the cold stupor point and below the threshold of activity. The intervals between $\mathrm{CO}_{2}$ bursts were 20-30 minutes after the measurements had lasted 3-4 hours and the bumblebees had fully recovered from the handling stress (Fig. 1). After every $\mathrm{CO}_{2}$ burst a characteristic peak followed, obviously due to abdominal contraction (Fig. 2). At $0^{\circ} \mathrm{C}$ the gas exchange was continuous.

\section{Abdominal pumping movements}

We regarded vigorous abdominal contractions as abdominal pumping movements. These visually well observable movements in bumblebee workers were always associated with discontinuous gas exchange cycles and the tracheae were seemingly actively ventilated by these movements. Usually the pumping movements started with the beginning of a $\mathrm{CO}_{2}$ cyclic release

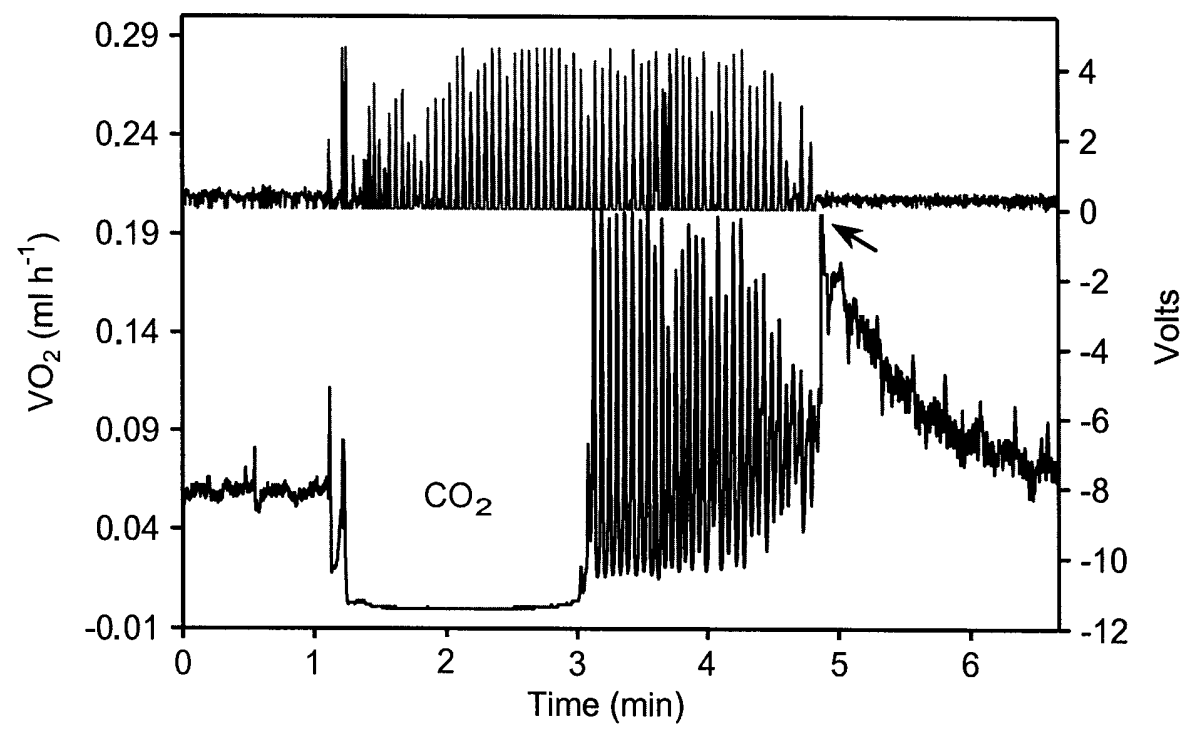

Fig. 2. A detail of Fig. 1. An optical recording (upper trace, Volts) and a simultaneous respirogram (lower trace) represent a typical example of when the onset of abdominal pumping movements (spikes) coincides with the beginning of a $\mathrm{CO}_{2}$ burst in a bumblebee. An arrow indicates a stroke of vigorous abdominal contraction, after which clear signals of abdominal pulsations followed. 


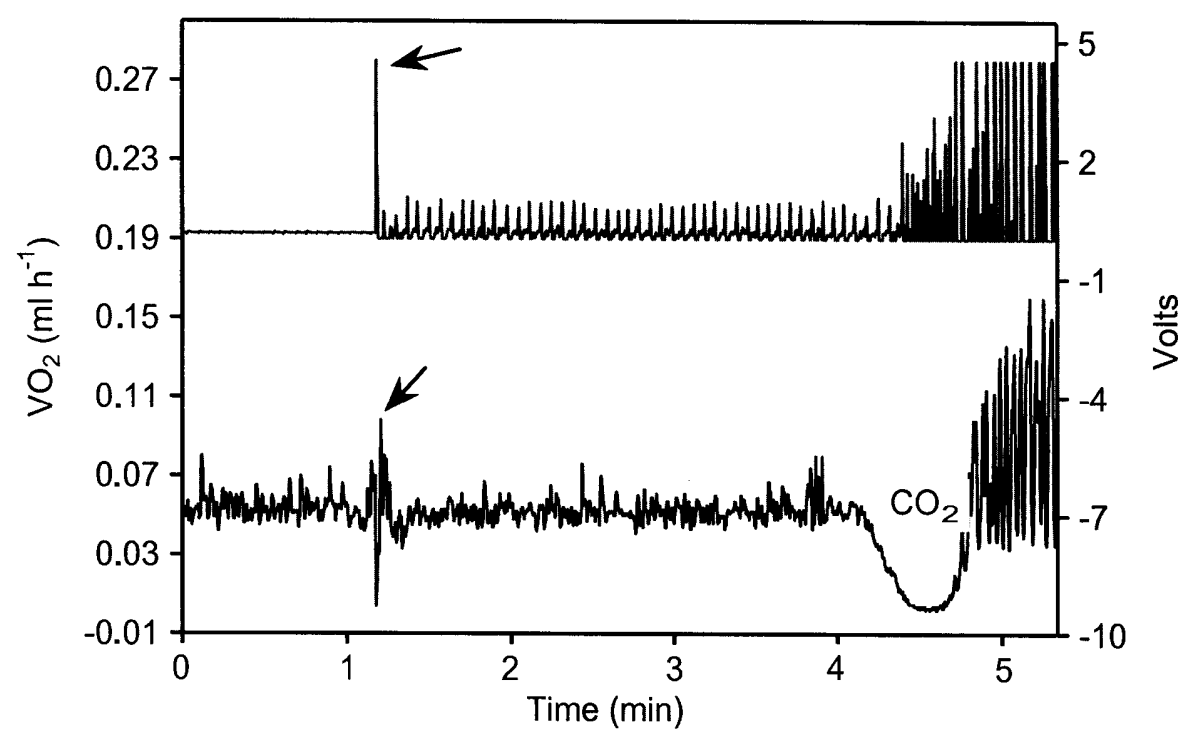

Fig. 3. A detail of Fig. 1 where the heart pause was followed by a period of heart activity (arrow) (upper trace). During the $\mathrm{CO}_{2}$ burst, peaks are seen due to vigorous pumping movements (lower trace).

(Fig. 1 and 2), but sometimes the pumping movements began at the end of the $\mathrm{CO}_{2}$ burst. The period of pumping lasted 2-4 minutes. About $20 \%$ of the bursts of an individual were not associated with abdominal pumping. There were also bumblebee individuals whose DGCs never concurred with bouts of vigorous abdominal pumping. The common frequency of the abdominal pumping movements was $0.5-0.7 \mathrm{~Hz}$.

The metabolic rate during the vigorous pumping movements was 2-3 times higher than the metabolic rate during the pumping pause $\left(0.6-1.2 \mathrm{ml} \mathrm{g}^{-1} \mathrm{~h}^{-1}\right.$ and $0.2-0.5$ $\mathrm{ml} \mathrm{g}^{-1} \mathrm{~h}^{-1}$, respectively).

\section{Abdominal pulsations}

The clear rhythmic signals with relative low amplitudes we regarded as "abdominal pulsations", as they were externally imperceptible even under a stereomicroscope.
Abdominal pulsations were easily seen on the respirometric recording after the ending of the vigorous pumping movements (Fig. 2). These pulsations caused rhythmic air pressure pulses inside the respirometer. The patterns of abdominal pulsations were often mixed with other signals.

\section{Heartbeats}

On the recordings of the respirometer and IR device, several signals were mixed with those of heartbeats. There were periods of very regular signals of low amplitudes, seen well only on the recordings of IR device (Fig. 1, 3). The simultaneous IR irradiation through the abdominal second and fifth segments showed the clear peristaltic nature of these movements, which were recognized as forward directed heartbeats (Fig. 4). We concluded that these weak heartbeat signals were recorded

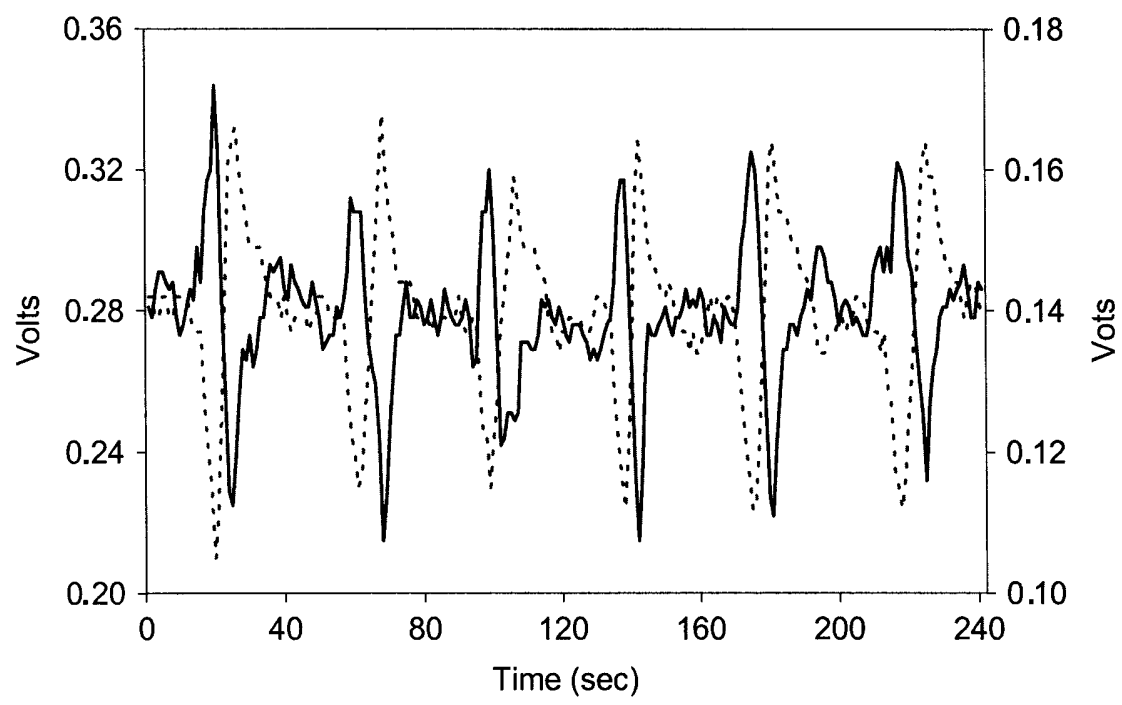

Fig. 4. The simultaneous records of IR-sensor diodes at the second (solid line) and fifth abdominal tergites (dotted line) during "heart pause", showing the forward directed heartbeats. Systolic contraction is down and diastole is up. This optical recording was made by means of high-grade amplification. 


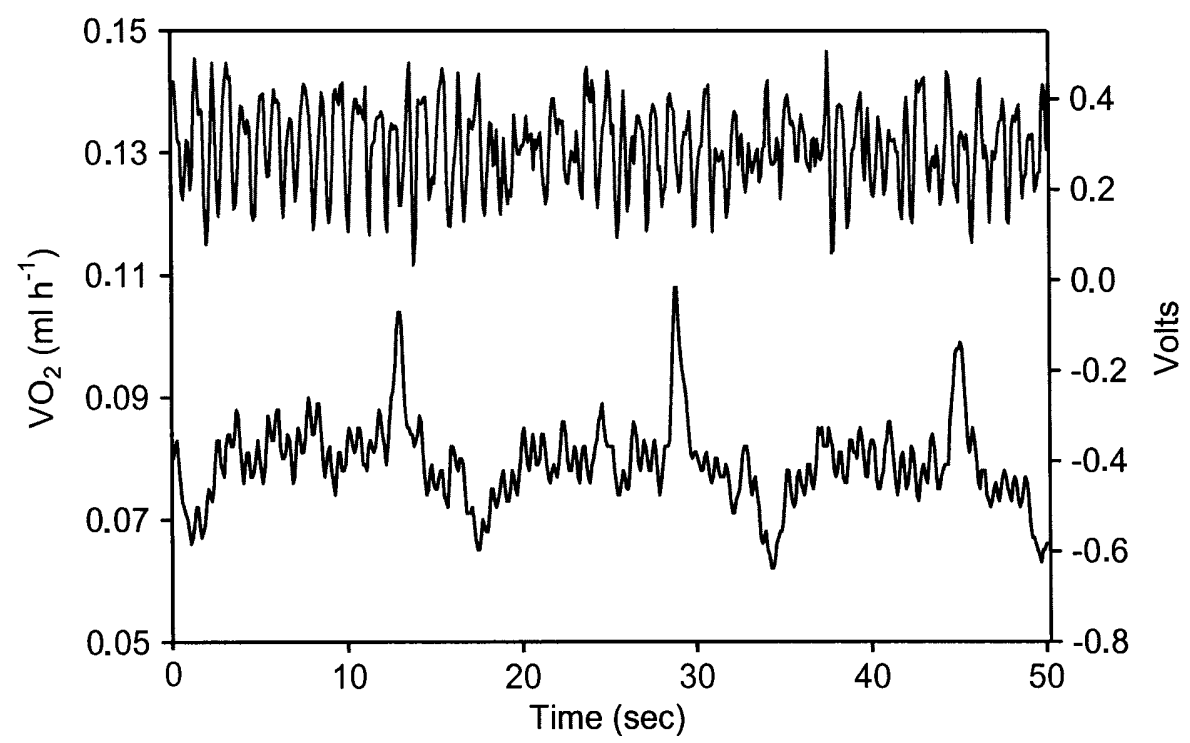

Fig. 5. A recording of an IR-sensor diode at the thorax side (upper trace), showing the contractions of wing muscles at $5^{\circ} \mathrm{C}$. A simultaneous respirogram (lower trace) with three abdominal pulses.

during the "heart pause", because after the pause these signals transformed into peaks of the same frequency, but of much higher amplitudes (Fig. 3). By our methods we could not determine the time when the "heart pause" ended. Abdominal pumping movements occurring during the $\mathrm{CO}_{2}$ cyclic release (Fig. 1) suppressed the weaker signals, including possible heartbeats which were mixed with the pumpings. The usual frequency of the supposed heartbeats was $0.25-0.3 \mathrm{~Hz}$ at $5^{\circ} \mathrm{C}$.

The heartbeat frequency of some bumblebee workers was similar to that of the abdominal pulsations, and sometimes a strict synchronization occurred between these two events. The amplitude of ventilation strokes was commonly 4-5 times higher than that of heartbeats. Thus it was evident that our cardiographic method allowed us to record the heartpulses only during the periods when other body movements were not mixed with heartbeats.

\section{Shivering}

By means of the IR optical device we recorded movements in the thorax with the frequency of $1.3-1.6 \mathrm{~Hz}$ at $5^{\circ} \mathrm{C}$, which we interpreted as fibrillar weak "shivering" of wing muscles (Fig. 5). The signals of the supposed shivering were sometimes superimposed on the signals of more vigorous muscular contractions with lower frequency. We recorded a clear shivering with a frequency of $5-7 \mathrm{~Hz}$ in the stressed bumblebee foragers at $10^{\circ} \mathrm{C}$ (Fig. 6).

\section{DISCUSSION}

In bumblebees rhythmic movements of different origin may occur: e.g., heartbeats, abdominal pumping movements and contractions of ventral diaphragm (Heinrich, 1976, 1994). In the present work some of the ordinary

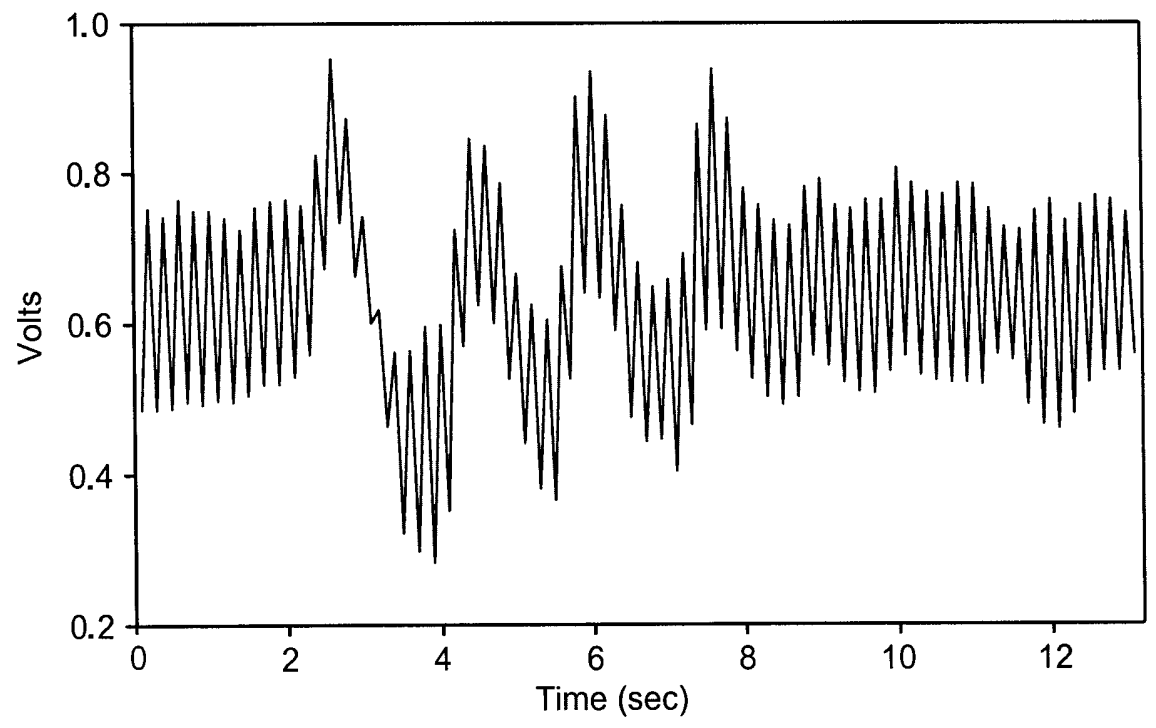

Fig. 6. An IR-sensor recording of the "shivering" of wing muscles of a bumblebee forager at $10^{\circ} \mathrm{C}$. The signals of muscular vibration are modulated by four strokes of abdominal movements. 
rhythmic movements were identified in the foragers of $B$. terrestris.

In some Lepidoptera and Hymenoptera haemolymph oscillation has been described, which works as a mechanism for the ventilation of tracheae and airsacs (Wasserthal, 1976, 1980, 1981, 1982). In sleeping $A$. mellifera the abdominal contractions coincided with the moment of transition from a long heartbeat pause to a prolonged beating of the heart which is always forward (Wasserthal, 1996). The backward flow of haemolymph through the perineural sinus happened mainly during the heart pause.

In the adult bumblebee of Bombus vosnessenskii and $B$. edwardsit, the ventilation movements are highly coordinated with the circulating actions of the dorsal vessel and ventral diaphragm, acting as a mechanism of thermoregulation (Heinrich, 1977). In the overheated bumblebees single abdominal pumping movements were synchronized with heart pulses, from which it could be suggested that the pressure evoked by abdominal contractions assisted the pumping movements of the heart (Heinrich, 1994).

The methods we used allowed us to record and recognize some rhythmic events of $B$. terrestris. These were first of all the discontinuous gas exchange cycles and vigorous abdominal pumping movements, which occurred during the $\mathrm{CO}_{2}$ bursts. We recorded periodically the very regular signals by IR sensors, which we interpreted as heartbeat pause with very weak heart pulsations.

In the present results $B$. terrestris showed a more or less regular alternation of periods of heartbeat with heart pauses, or rather with periods of very weak pulsations of the heart. No distinct coordination between the periods of heart activity and abdominal movements (pumping and pulsations) was found. However, the intermittent heart activity suggests haemolymph oscillation in $B$. terrestris at $5^{\circ} \mathrm{C}$, similar to that of $A$. mellifera and other bumblebee species described above but measured at higher temperatures.

We interpreted the very weak but regular movements caused by the abdomen as abdominal pulsations which are independent of muscular ventilation; with particular rhythms and frequencies. In our opinion the abdominal pulsations in $B$. terrestris are identical to the haemolymph extracardiac pressure pulses described by Slama (1976) and later found in the insects of various developmental stages and systematic groups (Slama, 1984, 1988). Thoracic ganglia regulate the extracardiac pressure pulses, and these pulses have a role to play in respiration and circulation (Slama, 1994, 1999, 2000). The extracardiac pressure pulses are independent of heartbeats, but these pressure pulses occurred during the $\mathrm{CO}_{2}$ bursts, as described during each burst in cecropia pupae (Slama, 1988) and in adult Periplaneta (Slama, 1999).

The vibrating of wing muscles or "shivering" was commonly recorded by wire electrodes as the action potentials of the fibrillar muscles; i.e., by an electrophysiological method (see Kammer \& Heinrich, 1972, 1974). The shivering in Manduca sexta was studied by tracheal pressure measurements (Wasserthal, 2001). The infrared irradiation method allowed us to measure the frequency and amplitude of the shivering in bumblebee workers in a contact-free manner.

ACKNOWLEDGEMENTS. The research was supported by Grants from the Estonian Science Foundation, No. 4116, No. 5330, No. 4993 and No. 4075.

\section{REFERENCES}

Befkman M. \& van Stratum P. 1999: Respiration in bumblebee queens: effect of life phase on the discontinuous ventilation cycle. Entomol. Exp. Appl. 92: 295-298.

HADLEY N.F. 1994: Ventilatory patterns and respiratory transpiration in adult terrestrial insects. Physiol. Zool. 67: 175-189.

HeINRICH B. 1976: Heat exchange in relation to blood flow between thorax and abdomen in bumblebees. J. Exp. Biol. 64: 561-585.

HeInRICH B. 1977: The physiology of exercise in the bumblebee. Am. Sci. 65: 455-465.

HeINRICH B. 1994: Der Hummelstaat. List Verlag, München, Leipzig, $318 \mathrm{pp}$.

KAMMER A.E. \& Heinrich B. 1972: Neural control of bumblebee fibrillar muscle during warm-up, stabilization of thoracic temperature and flight. J. Exp. Biol. 58: 677-688.

KAMmer A.E. \& HeinRich B. 1974: Metabolic rates related to muscle activity in bumblebees. J. Exp. Biol. 61: 219-227.

KeSTLER P. 1984: Respiration and respiratory water loss. In: Hoffmann K.H. (ed.): Environmental Physiology and Biochemistry of Insects. Springer, Berlin, pp. 137-183.

Kưusir A., Hitesaar K., Metspalu L. \& Tartes U. 1991: Gas exchange rhythms of Galleria mellonella (Lepidoptera: Pyralidae). Proc. Estonian Acad. Sci. Biol. 40: 145-156.

Kuusik A., Harak M., Hitesaar K., Metspalu L. \& Tartes U. 1996: Different types of external gas exchange found in pupae of greater wax moth Galleria mellonella (Lepidoptera: Pyralidae). Eur. J. Entomol. 98: 23-35.

Lighton J.R.B. \& Lovegrove B.G. 1990: A temperatureinduced switch from diffusive to convective ventilation in the honeybee. J. Exp. Biol. 154: 509-516.

Lighton J.R.B. 1994: Discontinuous ventilation in terrestrial insects. Physiol. Zool. 67: 142-162.

Rothe U. \& Nachtigall W. 1989: Flight of the honeybee IV Respiratory quotients and metabolic rates during sitting, walking and flying. J. Comp. Physiol. B 158: 739-749.

Silvola J. 1984: Respiration and energetic of the bumblebee Bombus terrestris queen. Holarctic Ecol. 7: 177-181.

SNyder G.K., Sheafor B., Scholnick D. \& Farrelly C. 1995: Gas exchange in the insect tracheal system. J. Theor. Biol. 172: 199-207.

SLAMA K. 1976: Insect haemolymph pressure and its determination. Acta Entomol. Bohemoslov. 73: 65-75.

SLAMA K. 1984: Recording of haemolymph pressure pulsations from the insect body surface. J. Comp. Physiol. (B) 154: 635-643.

Slama K. 1988: A new look at insect respiration. Biol. Bull. Mar. Biol. Lab. 175: 289-300.

SLAMA K. 1994: Regulation of respiratory acidemia by the autonomic nervous system (Coelopulse) in insects and ticks. Physiol. Zool. 67: 163-174.

Slama K. 1999: Active regulation of insect respiration. Ann. Entomol. Soc. Am. 92: 916-929.

SLAMA K. 2000: Extracardiac versus cardiac haemocoelic pulsation in pupae of the mealworm (Tenebrio molitor L.). $J$. Insect Physiol. 46: 977-992. 
Tartes U., KuUsiK A. \& Vanatoa A. 1999: Diversity in gas exchange and muscular activity patterns in insect studied by respirometer-actograph. Physiol. Entomol. 24: 150-157.

Tartes U., Kuusik A., Hiiesaar K., Metspalu L. \& Vanatoa A. 2000: Abdominal movements, heartbeats and gas exchange in pupae of the Colorado potato beetle, Leptinotarsa decemlineata. Physiol. Entomol. 25: 151-158.

WASSERTHAL L.T. 1976: Heartbeat reversal and its coordination with accessory pulsatile organs and abdominal movements in Lepidoptera. Experientia 32: 577-578.

WASSERTHAL L.T. 1980: Oscillating haemolymph "circulation" in the butterfly Papilio machaon L. revealed by contact thermography and photocell measurements. J. Comp. Physiol. 139: $145-163$.
WasserThal L.T. 1981: Oscillating haemolymph "circulation" and discontinuous tracheal ventilation in the giant silk moth Attacus atlas L. J. Comp. Physiol. B 145: 1-15.

WASSERTHAL L.T. 1982: Wechselseitige funktionelle und strukturelle Anpassungen von Kreislauf- und Tracheensystem bei adulten Insekten. Verh. Dtsch. Zool. Ges. 1982: 105-1 16.

WASSERTHAL L.T. 1996: Interaction of circulation and tracheal ventilation in holometabolous insects. Adv. Insect Physiol. 26: 297-351.

WASSERTHAL L.T. 2001. Flight-motor-driven respiratory air flow in the hawkmoth Manduca sexta. J. Exp. Biol. 204: 2209-2220.

Received November 9, 2001; revised February 28, 2002, accepted April 29, 2002 